\title{
Security Enhancement in Image Watermarking Using Combined Medium Sub-Band Wavelet Approach for Copyright Protection
}

\author{
Sridhar Balakrishnan* \\ Department of Electronics and Communication Engineering, MLR Institute of Technology, Hyderabad, India \\ Received 23 February 2020; received in revised form 24 February 2021; accepted 25 February 2021 \\ DOI: https://doi.org/10.46604/peti.2021.5316
}

\begin{abstract}
Watermarking in multimedia content has secured attractive approaches in research society since nowadays the transmission of digital content in wireless medium has been enormous. Along with the distribution of digital content, it is important to claim ownership. A non-blind detection technique is proposed in this paper. First, an original image undergoes the wavelet decomposition such as LL1, LH1, HL1, and HH2. Afterward, the medium level sub-band coefficients of the image are subject to the comfortable shares for merging. Next, one of the shares from the LH1 band is merging with another one of the shares in HL1 band. In this stage, the copyright mark is fetched into the merged sub-band coefficients. Finally, an inverse wavelet transform is applied to receive the watermarked image. To prove the authentication, the original image and the watermarked image undergo the same operation and acquire copyright information. Experimental results achieve that the proposed approach can withstand various image processing attacks.
\end{abstract}

Keywords: copyright protection, image sharing, sub-band fusion, wavelet transform, watermarking

\section{Introduction}

Digital data possess the characteristics of making multiple copies without any difference. In the fast-growing wireless communications, a huge number of digital information is transmitted every second. The third party can easily acquire and claim ownership of the information. Consequently, the protection of licensed innovation is a critical thought for the present world [1-2]. Watermarking in digital contents is used to protect the multimedia information [3-6]. The watermarking technology has developed rapidly in the present era, and has given rise to the exponential growth of research findings in the field of copyright marking over the years. In a digital image watermarking system, the data to be embedded is called the digital copyright image, and the image where the watermark image to be embedded is called the host image.

Digital image watermarking can be classified based on the domain in which the data fetching processes can be carried out. The two major domains in watermarking technology are spatial and transform domains. Least significant bits (LSB) belongs to the spatial domain [7]; discrete Fourier transform (DFT) [8], discrete cosine transform (DCT) [9], and discrete wavelet transform (DWT) belong to the transform domain [10]. Watermarking in the spatial domain is represented by modifying spatial characteristics directly, such as pixel values. On the other hand, watermarking in the transform domain converts images to the frequency signal first and then modifies the coefficient of certain frequencies.

The Singular Value Decomposition (SVD) is a well-known technique for factorizing a rectangular matrix, real or complex, which has been widely employed in the image processing application such as image compression, face recognition, watermarking and texture classification. Robust copyright marking approach should withstand the identity data under different

* Corresponding author. E-mail address: sridharbece@gmail.com

Tel.: +91-9894173468 
image processing attacks, including filtering, compression, and cropping. Robustness, capacity, and imperceptibility [11] are the three characteristics of watermarking system, but all watermarking approaches only satisfy two characteristics. The rest of the manuscript is formed as follows: Related work is highlighted in section II. The proposed approach and the experimental results are discussed in sections III and IV. The conclusion is placed in section V.

\section{Related Works}

The extensive literature gathered and related to image watermarking techniques is critically inspected and exhibited in this section. Further, the summary of the literature review is also furnished at the end of the section. Lin et al. [12] developed a marking system under DWT. This algorithm performs the DWT on the original image and fetches the watermark into the HL and LH sub-bands. The experimental results demonstrate that the developed technique yields better results than the previous methodologies in terms of the quality of the watermarked image, the recovered watermark with or without attack, and time efficiency. The experiment results reported in the paper also suggest that this method can achieve ownership protection.

A novel technique was invented for robust wavelet-based watermarking [13]. This approach addresses the way to fetch signature data in wavelet transform coefficients at the selected group, varying the watermark strength according to the range of sub-band information so that the group can be enabled wherever the respective coefficients reside. At first, the original image can be decomposed into four levels by DWT. Next, the edges of the sub-band can be identified by the Sobel edge detector. The pipeline of the copyright is carried out through the sub-band coefficients that lie on edges, and in that zone the level of the disturbance is less noticeable, with a sub-band level-dependent strength. In addition to that, there are only a few coefficients ready to embed the watermark around the corners, utilizing a distinct strengthening factor captured by a morphological dilation operation.

Mohammed and Sidqi [14] clarified a multi-band wavelet-based image watermarking approach. Right now, a versatile strategy is created depending on the selectivity parameter of band coefficients for hiding the watermark. The benefits of this methodology are using a large number of wavelet groups during the inserting procedure. Surekha \& Sumathi [15] examined the copyright protection by using an image-based discrete wavelet transform through genetic algorithm. The scaling variable of the copyright is the most vital parameter that enhances the visual straightforwardness and strength against attacks.

Abdallah et al. [16] proposed a blind watermarking technique under wavelet transform, by which an identity mark is fetched into the coarsest scale wavelet coefficients. The image undergoes the three-level decomposition and the copyright mark is installed into the coarse scale. Sood and Goyal [17] proposed the Genetic Algorithm (GA)-Bacterial Foraging Algorithm (BFO) and DWT for watermarking. In this method, the five-level DWT is utilized in the spatial transform. Yahya et al. [18] developed an algorithm based on discrete wavelet transform to embed a watermark in the selected coefficient blocks only. For the extraction of copyright, a probabilistic neural network is employed. The experimental result demonstrates that the proposed approach provides copyrighted images with a high degree of imperceptibility and robustness to common attacks. From the above reviews, it is clear that we cannot install much data into the host image under frequency domain because the quality of the original image may be degraded significantly. Spatial domain provides successful recovery of watermark at receiver end, but the robustness is very limited. Hence the identification of marking a region of copyright data plays a vital role, and our proposed approach gives the direction to implant a watermark into the merged sub-bands coefficients under wavelet. The remaining section gives the proposed approach, and follows with experimental results and the conclusion.

\section{Proposed Approach}

The watermarking technique under wavelet is used positively for concealing the copyright information to achieve imperceptibility in digital content. Excellent spatial localization and multi-resolution are remarkable characteristics of 
wavelets. In our proposed approach, Daubechies wavelets are enforced for the decomposition of the original image because the wavelets are orthonormal and compactly supported scaling and wavelet functions. Initially original gray image $[Y]_{m \times n}$ is subject to the wavelet transformation such as $L L_{l}, L H_{l}, H L_{l}$ and $H H_{l}$. Fig. 1 shows the procedure of proposed approach.

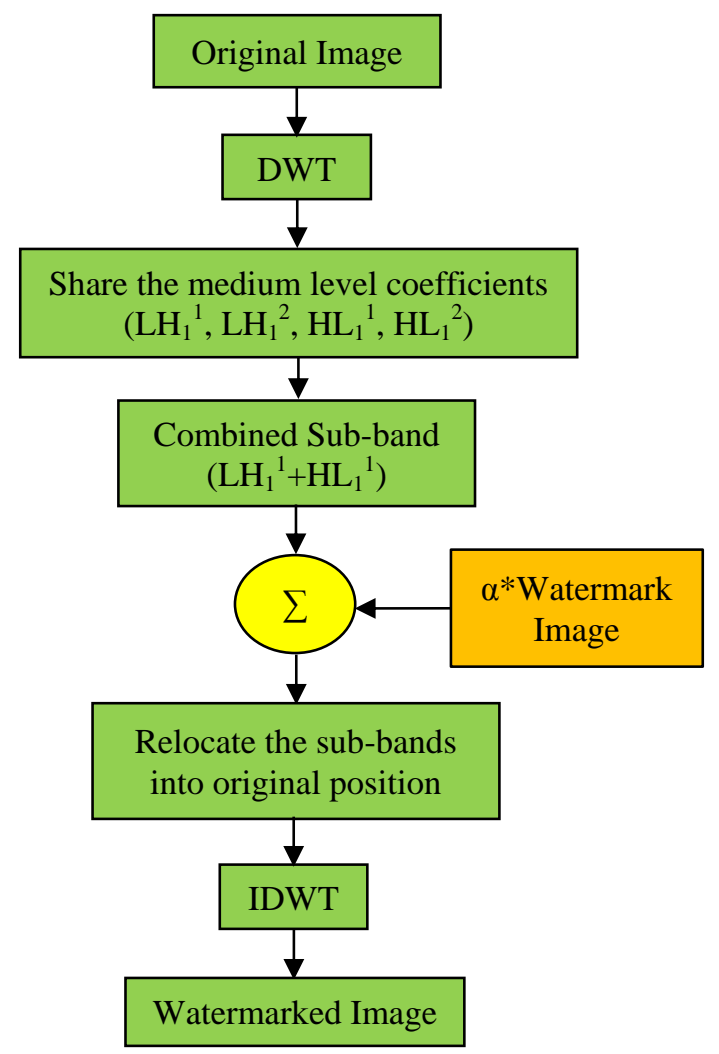

Fig. 1 Work flow of proposed approach

$Y \stackrel{D W T}{\longrightarrow}\left(\begin{array}{llllllll}L L_{l} & L L_{l} & L L_{l} & L L_{l} & L H_{l} & L H_{l} & L H_{l} & L H_{l} \\ L L_{l} & L L_{l} & L L_{l} & L L_{l} & L H_{l} & L H_{l} & L H_{l} & L H_{l} \\ L L_{l} & L L_{l} & L L_{l} & L L_{l} & L H_{l} & L H_{l} & L H_{l} & L H_{l} \\ L L_{l} & L L_{l} & L L_{l} & L L_{l} & L H_{l} & L H_{l} & L H_{l} & L H_{l} \\ H L_{l} & H L_{l} & H L_{l} & H L_{l} & H H_{l} & H H_{l} & H H_{l} & H H_{l} \\ H L_{l} & H L_{l} & H L_{l} & H L_{l} & H H_{l} & H H_{l} & H H_{l} & H H_{l} \\ H L_{l} & H L_{l} & H L_{l} & H L_{l} & H H_{l} & H H_{l} & H H_{l} & H H_{l} \\ H L_{l} & H L_{l} & H L_{l} & H L_{l} & H H_{l} & H H_{l} & H H_{l} & H H_{l}\end{array}\right)$

$W=\left(\begin{array}{ccccc}w_{l, 1} & w_{1,2} & \cdot & \cdot & w_{1, n} \\ w_{2,1} & w_{2,2} & & & w_{2, n} \\ \cdot & & \cdot & \cdot \\ \cdot & & & \cdot & \cdot \\ w_{m, 1} & w_{m, 2} & \cdot & & w_{m, n}\end{array}\right)_{r X c}$

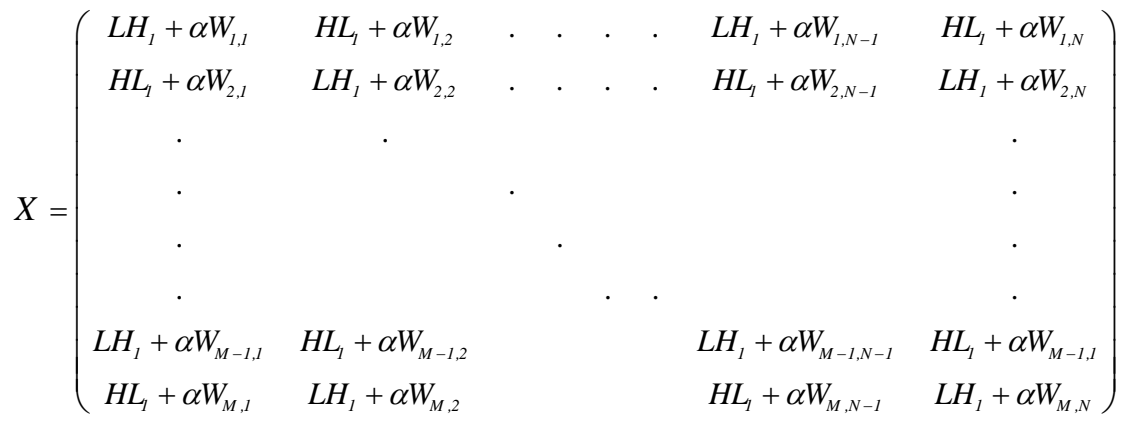




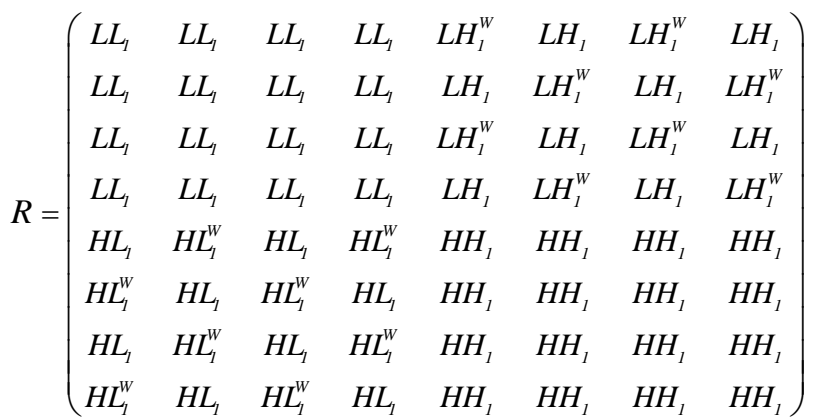

$$
\begin{aligned}
& \left(\begin{array}{cc}
L L_{1} & L H_{1}^{W} \\
H L_{1}^{W} & H H_{1}
\end{array}\right) \stackrel{I D W T}{\longrightarrow} Y^{W} \\
& {\left[Y^{W}\right]_{m x n} \stackrel{D W T}{\longrightarrow}\left[\begin{array}{cc}
L L_{R} & L H_{R}^{W} \\
H L_{R}^{W} & H H_{R}
\end{array}\right]_{m \times n}} \\
& W=\frac{\left(L H_{R}^{1}+H L_{R}^{1}\right)-\left(L H_{1}^{l}+H L_{l}^{l}\right)}{\alpha}
\end{aligned}
$$

Secret image sharing has received considerable attention in recent years. Secure assurance of images is taken as a primary concern in military or commercial based applications. In our proposed framework, the original image is grouped into alternative pixels shares. Next, the sub-band coefficients are merged, and finally, normal marked structures can be obtained by combining the allotments into a single image. What follows is the proposed algorithm:

\begin{tabular}{|l|}
\hline Algorithm 1: Image Sharing \\
\hline Begin: \\
Read the Rows do \\
Read the Columns 2 do \\
if mod $(i, 2) \neq 0$ \\
First share (odd, even) \\
else \\
First share (even, odd) \\
endif \\
end \\
end \\
Second share= Original Image-First share \\
End
\end{tabular}

\section{Experimental Results}

This combined sub-band based marking approach is executed using MATLAB. For installing the identity information, we utilize a cameraman image of size $256 \times 256$ and the copyright image of size $32 \times 32$. The original image, the combined sub-bands image, the watermark image, and the watermarked image are displayed in Fig. 2.

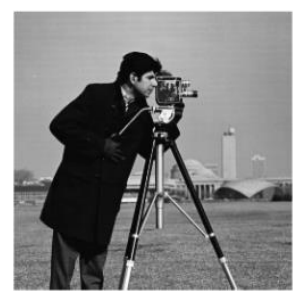

(a) Original image

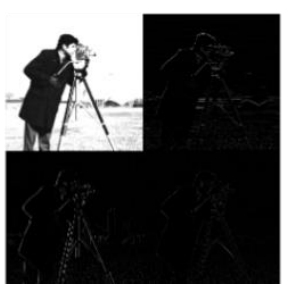

(b) DWT of an image

Fig. 2 Experimental results of proposed approach 


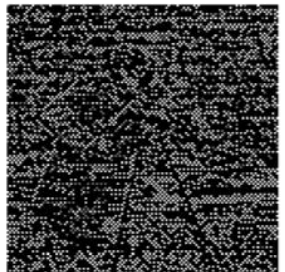

(c) Combined share of medium level sub-band image

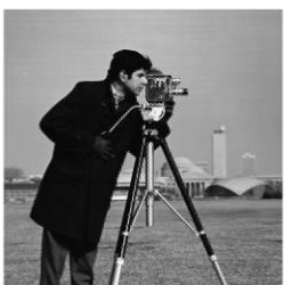

(e) Watermarked image

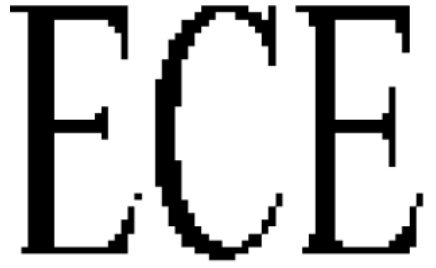

(d) Watermark image

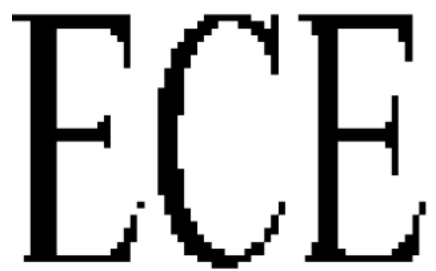

(f) Extracted watermark

Fig. 2 Experimental results of proposed approach (continued)

\subsection{Invisibility test}

The validation of the method is concentrated on the invisibility of the embedding data it generates. To estimate the imperceptibility, Mean Square Error (MSE) and Peak signal to noise ratio (PSNR) are the basic parameters. The difference between the original image $\mathrm{O}(\mathrm{i}, \mathrm{j})$ and the watermarked image W $(\mathrm{i}, \mathrm{j})$ is MSE. The average MSE of this proposed scheme is 0.0012. PSNR is used to justify the degree of excellence of the watermarked image. The PSNR of our proposed approach is $61.7259 \mathrm{~dB}$. The formulas of MSE and PSNR are expressed as

$$
\begin{aligned}
& M S E=\frac{1}{X Y} \sum_{i, j}(O(i, j)-W(i, j))^{2} \\
& P S N R=10 \log _{10}\left(\frac{255^{2}}{M S E}\right)
\end{aligned}
$$

The correlation co-efficient is another parameter to find out the robustness of the techniques against the image processing attacks.

$$
r=\frac{\sum_{x} \sum_{y}\left(O_{x y}-\bar{O}\right)\left(E_{x y}-\bar{E}\right)}{\sqrt{\left(\sum_{x} \sum_{y}\left(O_{x y}-\bar{O}\right)^{2}\right)\left(\sum_{x} \sum_{y}\left(E_{x y}-\bar{E}\right)^{2}\right)}}
$$

The correlation coefficient value may be one or zero, depending on whether the watermarked image and the original image are identical or not. Eq.10 shows the formula to generate the correlation coefficient between two images.

\subsection{Attacks in image watermarking}

The most common attacks of image watermarking are noise attacks, geometrical attacks, and filtering attacks. In our method, different noises (e.g. Salt \& pepper, Speckle, Gaussian, and Poisson with default noise density) are introduced in a watermarked image with default noise density and the robustness of the system is measured.

Also, some additional evaluation of median filtering and low pass filtering attack on the watermarked image and measure the performance of the algorithm. Table 1 demonstrates the PSNR values between DWT and SVD. Table 2 shows the performance of an algorithm with different attacks. 
Table 1 PSNR values of the proposed approach with different bands

\begin{tabular}{|c|c|c|}
\hline Scaling & DWT & SVD \\
\cline { 2 - 3 } Factor & PSNR(dB) & PSNR(dB) \\
\hline 0.01 & 74.8455 & 68.8240 \\
\hline 0.02 & 68.8249 & 62.8034 \\
\hline 0.03 & 65.3031 & 59.2815 \\
\hline 0.04 & 62.8043 & 56.7828 \\
\hline 0.05 & 60.8661 & 54.8446 \\
\hline 0.06 & 59.2825 & 53.2609 \\
\hline 0.07 & 57.9435 & 51.9220 \\
\hline 0.08 & 56.7837 & 50.7622 \\
\hline 0.09 & 55.7607 & 49.7391 \\
\hline 0.1 & 54.8455 & 48.8240 \\
\hline
\end{tabular}

Table 2 Performance of the proposed approach

\begin{tabular}{|c|c|c|}
\hline \multirow{2}{*}{ Attacks } & $\begin{array}{c}\text { Watermarked } \\
\text { Image }\end{array}$ & $\begin{array}{c}\text { Watermark } \\
\text { Image }\end{array}$ \\
\cline { 2 - 3 } & PSNR $(\mathrm{dB})$ & PSNR $(\mathrm{dB})$ \\
\hline No Attacks & 61.7259 & 43.9923 \\
\hline Salt \& Pepper & 56.3432 & 36.1278 \\
\hline Speckle & 53.4509 & 36.2309 \\
\hline Gaussian & 45.7534 & 35.3267 \\
\hline Poisson & 37.0921 & 34.7877 \\
\hline Median Filtering & 38.7204 & 37.9241 \\
\hline Low pass filtering & 39.4012 & 39.3809 \\
\hline
\end{tabular}

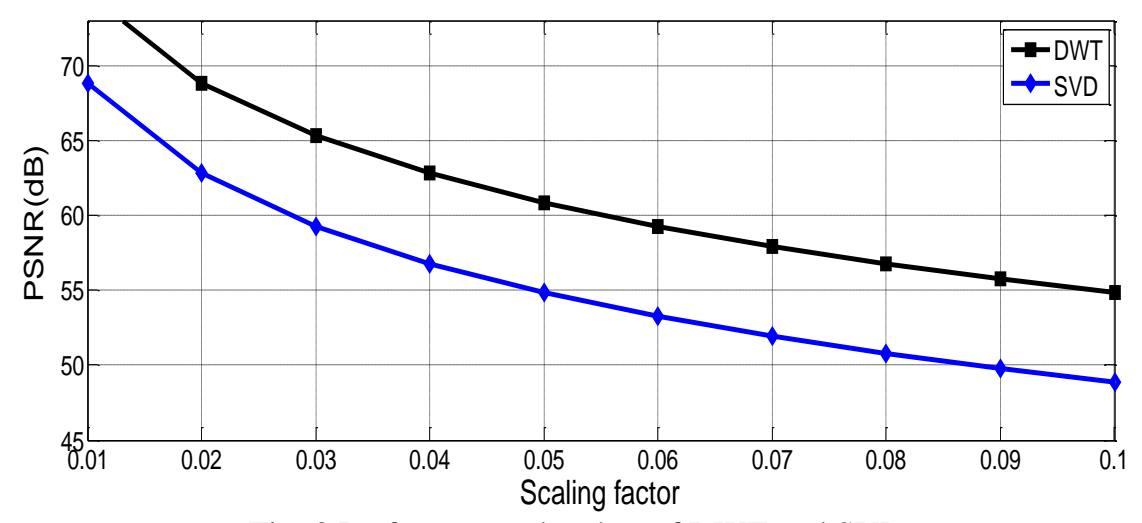

Fig. 3 Performance sketches of DWT and SVD

The above Fig. 3 represents the performance curve between the DWT and SVD. In this noted that DWT based watermarking systems hold better PSNR value than the SVD. Also, this proposed approach has secured a higher PSNR value than $37.676 \mathrm{~dB}$ (the value obtained by Tanwar and Khurana [19]).

\section{Conclusions}

Digital image watermarking innovation has been drawing the attention of researchers and experts as another technique for ensuring copyright for digital images. It is acknowledged by installing a make-able data which is imperceptible to the human visual systems, and it is called a watermark. Henceforth watermarking in images is the process in which a discrete data embedded within an image, imposing unperceived modifications on the image. In this paper, the sub-band sharing based methodology is exhibited. Here, the copyright information is covered into the combined wavelet coefficients; henceforth the level of robustness is increased. In this approach, PSNR and correlation coefficient is $61.7259 \mathrm{~dB}$ and 0.9985 respectively. The defined methodology acquires less complexity and high efficiency. 


\section{Conflicts of Interest}

Author declares no conflict of interest.

\section{References}

[1] Q. Li and I. J. Cox, "Using Perceptual Models to Improve Fidelity and Provide Resistance to Valumetric Scaling for Quantization Index Modulation Watermarking," IEEE Transaction on Information Forensics and Security, vol. 2, no. 2, pp. 127-139, June 2007.

[2] A. Piva, T. Bianchi, and A. De Rosa, "Secure Client-Side ST-DM Watermark Embedding," IEEE Transactions on Information Forensics and Security, vol. 5, no. 1, pp. 13-26, March 2010.

[3] A. Piva, F. Bartolini, and M. Barni, "Managing Copyright in Open Networks," IEEE Internet Computing, vol. 6, no. 3, pp. 18-26, May-June 2002.

[4] B. Sridhar, "A Wavelet Based Watermarking in Video Using Layer Fusion Technique," Pattern Recognition and Image Analysis, vol. 28, no. 3, pp. 537-545, July 2018.

[5] V. M. Potdar, S. Han, and E. Chang, “A Survey of Digital Image Watermarking Techniques,” 3rd IEEE International Conference on Industrial Informatics (INDIN), August 2005, pp. 709-713.

[6] Y. Wang, J. F. Doherty, and R. E. Van Dyck, "A Wavelet Based Watermarking Algorithm for Ownership Verification of Digital Image,” IEEE Transactions on Image Processing, vol. 11, no. 2, pp. 77-88, February 2002.

[7] S. Dey, A. Abraham, and S. Sanyal, “An LSB Data Hiding Technique Using Natural Number Decomposition,” Third International Conference on Intelligent Information Hiding and Multimedia Signal Processing, November 2007, pp. 473-476.

[8] G. Ren, C. Zhang, and X. Yang, "Blind Mesh Watermarking Based on the Featured Points in the Frequency Domain," Second International Workshop on Knowledge Discovery and Data Mining, January 2009, pp. 701-704.

[9] N. Hubballi and D. P. Kanyakumari, "Novel DCT Based Watermarking Scheme for Digital Image," International Journal of Recent Trends in Engineering, vol. 1, no. 1, pp. 430-433, 2009.

[10] B. Sridhar, "Cross-Layered Embedding of Watermark on Image for High Authentication," Pattern Recognition and Image Analysis, vol. 29, no. 1, pp. 194-199, January 2019.

[11] B. Sridhar, "A Wavelet Based Watermarking Approach in Concatenated Square Block Image for High Security,” Journal of Automation, Mobile Robotics and Intelligent Systems, vol. 12, no. 3, pp. 68-72, December 2018.

[12] H. J. Lin, C. W. Lu, and C. M. Chiang, "DWT-Based Watermarking Technique Associated with Embedding Rule," 10th WSEAS International Conference on Signal Processing, Computational Geometry and Artificial Vision, August 2010, pp. 23-28.

[13] J. N. Ellinas, “A Robust Wavelet-Based Watermarking Algorithm Using Edge Detection,” World Academy of Science, Engineering and Technology, vol. 1, no. 10, January 2007.

[14] A. A. Mohammed and H. M. Sidqi, "Robust Image Watermarking Scheme Based on Wavelet Technique," International Journal of Computer Science and Security (IJCSS), vol. 5, no. 4, pp. 394-404, September-October 2011.

[15] P. Surekha and S. Sumathi, "Implementation of Genetic Algorithm for a DWT Based Image Watermarking Scheme," ICTACT Journal on Soft Computing: Special Issue on Fuzzy in Industrial and Process Automation, vol. 2, no. 1, pp. 244-252, July 2011.

[16] H. A. Abdallah, M. M. Hadhoud, A. A. Shaalan, and F. E. Abd El-samie, "Blind Wavelet-Based Image Watermarking," International Journal of Signal Processing, Image Processing and Pattern Recognition, vol. 4, no. 1, pp. 15-28, 2011.

[17] S. Sood and A. Goyal, "Watermarking Relational Databases Using Genetic Algorithm and Bacterial Foraging Algorithm," International Journal of Information and Computation Technology, vol. 4, no. 17, pp. 1877-1884, 2014.

[18] A. N. Yahya, H. A. Jalab, A. Wahid, and R. M. Noor, "Robust Watermarking Algorithm for Digital Images Using Discrete Wavelet and Probabilistic Neural Network,” Journal of King Saud University-Computer and Information Sciences, vol. 27, no. 4, pp. 393-401, October 2015.

[19] P. Tanwar and M. Khurana, "Improved PSNR and NC in Digital Image Watermarking Using RDWT and SVD," International Journal of Advanced Research in Computer Science and Software Engineering Research, vol. 6, no. 5, pp. 955-959, May 2016.

Copyright $@$ by the authors. Licensee TAETI, Taiwan. This article is an open access article distributed under the terms and conditions of the Creative Commons Attribution (CC BY-NC) license (https://creativecommons.org/licenses/by-nc/4.0/). 